\title{
High predictability of the winter Euro-Atlantic climate from cryospheric variability
}

\author{
J. García-Serrano` and C. Frankignoul
}

\begin{abstract}
Seasonal prediction skill for surface winter climate in the Euro-Atlantic sector has been limited so far $^{1-3}$. In particular, the predictability of the winter North Atlantic Oscillation, the mode that largely dominates regional atmospheric and climate variability, remains a hurdle for present dynamical prediction systems ${ }^{4,5}$. Statistical forecasts have also been largely elusive ${ }^{6-8}$, but October Eurasian snow cover has been shown to be a robust source of regional predictability ${ }^{9,10}$. Here we use maximum covariance analysis to show that Arctic sea-ice variability represents another good predictor of the winter Euro-Atlantic climate at lead times of as much as three months. Cross-validated hindcasts of the winter North Atlantic Oscillation index using September sea-ice anomalies yield a correlation skill of 0.59 for the period 1979/1980-2012/2013, suggesting that $35 \%$ of its variance could be predicted three months in advance. This skill can be further enhanced, at the expense of a shorter lead time, by using October Eurasian snow cover as an additional predictor. Skilful predictions of winter European surface air temperature and precipitation are also obtained with September sea ice as the only predictor. We conclude that it is important to incorporate Arctic sea-ice variability in seasonal prediction systems.
\end{abstract}

The North Atlantic Oscillation (NAO) is the leading mode of atmospheric variability during North Atlantic-European winter ${ }^{11}$ (December-February) and it strongly influences the interannual variability of surface temperature and precipitation as it is associated with changes in the westerly flow reaching the continent from the ocean and a modulation of the North Atlantic storm track ${ }^{11}$. It also governs changes in extreme weather events ${ }^{12}$. Forecasting the NAO is thus of paramount importance for skilful predictions of European winter climate anomalies ${ }^{4}$.

The NAO is a dominant mode of intrinsic atmospheric variability $^{11}$ and atmospheric internal variability is strong during winter. This probably explains why winter is the season with the lowest prediction skill from persistence-based forecasts ${ }^{1}$ and yearto-year variations in the phase and amplitude of the winter NAO has often deemed to be largely unpredictable ${ }^{11}$. However, there is increasing evidence that the winter NAO is in part driven by slow changes in the Earth boundary conditions, which is indeed the premise for the feasibility of seasonal prediction ${ }^{3}$. The most explored boundary forcing has been the sea surface temperature, but its usefulness for forecasting purposes is $\operatorname{low}^{3,6,7}$. The progression of October snow-cover extent over Eurasia has been recently shown to provide a good NAO predictive skill ${ }^{9}$ and realistic snow initialization has a positive impact on dynamical prediction systems ${ }^{13-15}$. There have also been encouraging studies on the influence of autumn Arctic sea-ice concentration (SIC) on the winter Euro-Atlantic atmospheric circulation ${ }^{16-18}$. Here we use satellite-derived and re-analysed data to show that autumn Arctic SIC is a robust source of predictive skill for the winter NAO. This illustrates the relevance of cryospheric variability for skilful forecasts of surface winter climate in Europe and provides a benchmark correlation skill that dynamical prediction systems must aspire to reach or exceed.

To optimize the SIC pattern that most strongly leads the winter atmospheric circulation, we use maximum covariance analysis (MCA; ref. 7), which estimates the main modes of covariability between two fields and their respective time evolution (expansion coefficients) while making no a priori assumption on the spatial patterns. We consider Arctic SIC during autumnal months, September-November, and winter Euro-Atlantic sea level pressure (SLP) in the 1979/1980-2012/2013 period. Long-term trends are removed from each field to focus on interannual climate variability. Figure 1 shows the leading MCA mode for SIC in September, which explains $53 \%$ of the squared covariance fraction and yields the largest correlation $(0.73)$ between the expansion coefficients of SIC and SLP. This correlation reduces to 0.70 and 0.67 in the corresponding MCA analysis with October and November SIC, respectively. These differences in correlation are not statistically significant, but we focus on September sea ice (hereafter MCA-SIC) as it provides the longest lead time for predicting the winter Euro-Atlantic atmospheric circulation. The MCA-SLP expansion coefficient correlates at -0.98 with the winter NAO index, which means that the September sea-ice anomalies from MCA-SIC (Fig. 1a) precede a negative NAO phase in winter (Fig. 1b). Note that the SIC patterns are derived from the MCA-SIC expansion coefficient and thus slightly differ from the regressions onto the winter NAO index.

The MCA-SIC pattern shows negative anomalies (a sea-ice reduction corresponding to a retreat of the sea-ice edge) over the eastern Arctic from the Greenland Sea to the western Laptev Sea, with maximum amplitude over the northern Barents-eastern Kara seas. The SIC in the Pacific sector is also reduced over the Chukchi Sea-Bering Strait. These sea-ice anomalies resemble the autumn (September-November) patterns found in previous studies in relation to the winter Arctic Oscillation ${ }^{16,17}$. Furthermore, the MCA-SIC yields positive SIC anomalies (increased sea-ice extent) over the western Arctic, from the East Siberian Sea to the Canada Basin.

Regressing forward in time the MCA-SIC expansion coefficient onto October and November SIC anomalies illustrates the evolution of the September anomaly that precedes the winter NAO. As shown in Fig. 1c-d, the westward displacement of the largest anomalous sea-ice retreat follows the climatological expansion of sea-ice over the eastern Arctic (green contour). The October SIC regression map (Fig. 1c) indicates that negative anomalies expand over the Kara Sea, whereas the maximum sea-ice reduction (around -15\%) is located over the northern Barents Sea and the sea-ice retreat reaches farther 
a $\quad$ MCA-SIC (September) $\mathrm{scf}=53 \% r=0.73$

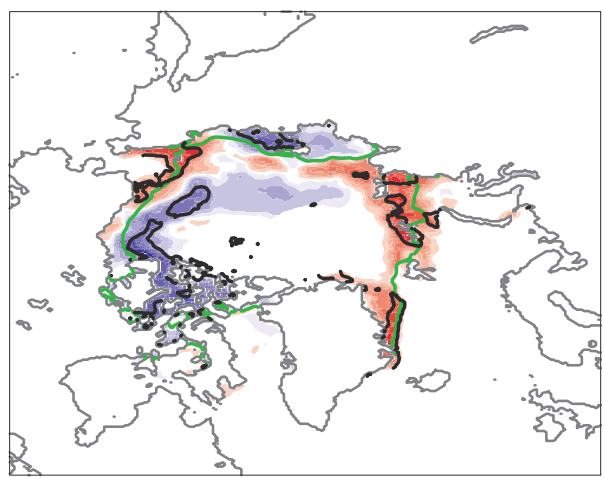

c

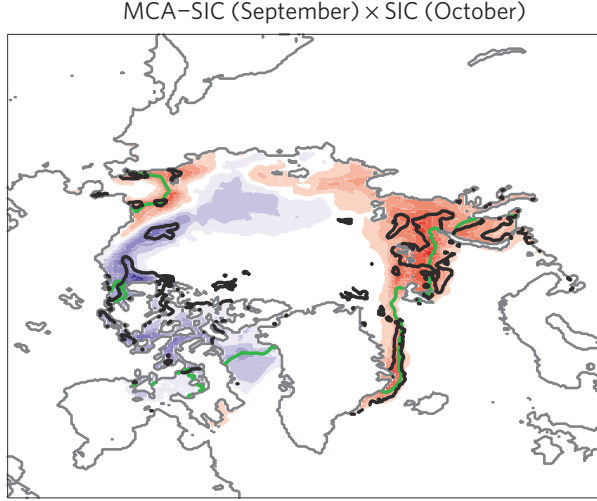

b

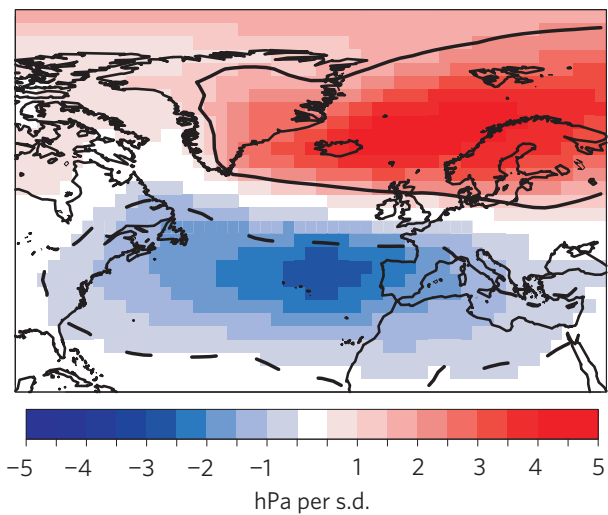

d

MCA-SIC (September) $\times$ SIC (November)

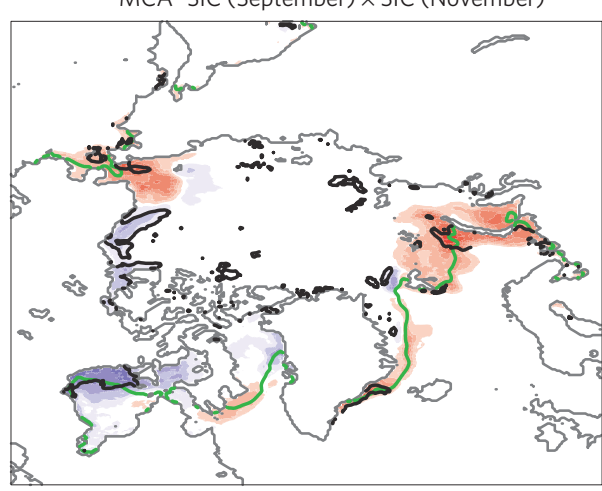

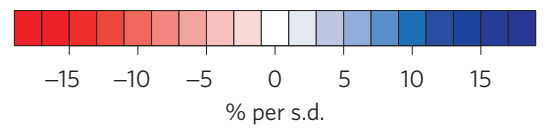

Figure 1 | September Arctic sea ice as the predictor field. a,b, Leading mode of covariability between detrended September Arctic SIC anomalies (predictor field) and winter SLP anomalies over the North Atlantic-European sector $90^{\circ} \mathrm{W}-40^{\circ} \mathrm{E}, 20^{\circ} \mathrm{N}-90^{\circ} \mathrm{N}$ (predictand field); the squared covariance fraction (scf) explained by the MCA mode and the correlation between expansion coefficients ( $r$ ) are indicated. DJF: December, January, February. $\mathbf{c}, \mathbf{d}$, Evolution of the September Arctic sea-ice pattern throughout October and November. The maps are displayed in terms of amplitude by regressing detrended SIC anomalies (\%; a,c,d) and SLP anomalies ( $\mathrm{hPa} ; \mathbf{b}$ ) on to the MCA-SIC expansion coefficient. Statistically significant areas at 95\% confidence level based on a two-tailed $t$-test are contoured. s.d., standard deviation.

south on the eastern coast of Greenland. In the western Arctic, the sea-ice expansion loses significance except along the Canadian coast and practically no significant SIC anomalies remain in the Pacific. In November (Fig. 1d), the Kara Sea is mostly frozen, so that the strong sea-ice reduction (larger than $-10 \%$ ) is seen only in the northeastern Barents Sea. The evolution of the SIC anomalies thus supports earlier results suggesting that the Barents-Kara region plays the main role in the September SIC influence on the winter NAO (ref. 19).

As the influence of the NAO on the European winter climate is so strong, predicting its state (that is, the value of the NAO index) in advance should strongly enhance the value of seasonal climate forecasts. The high correlation between the MCA expansion coefficients of SLP and SIC in September suggests that empirical predictions of the winter NAO index could be based on the MCA-SIC time series. To assess the potential predictability of this statistical model, a one-year-out cross-validation method ${ }^{20}$ was used. This method avoids artificial skill and allows estimation of the $95 \%$ prediction interval (Fig. 2, grey shading). The cross-validated hindcast of the NAO (Fig. 2a, red line) captures reasonably well the amplitude of the observed anomalies and rightly reproduces its lowfrequency variability during the period $1979 / 1980-2012 / 2013$. The winter of 2011/2012 is the only year in which the observed NAO index (Fig. 2, black line) does not fall within the predictive range.
The cross-validated NAO correlation skill is 0.59 , which indicates that September SIC could explain as much as $35 \%$ of the year-toyear variance of the winter NAO three months in advance. Using October or November SIC would lead to a similar, not statistically different, NAO prediction skill of 0.61 and 0.58 , respectively.

To illustrate the potential predictability of the winter surface temperature and precipitation in Europe, a statistical prediction model at grid-point level was designed by using MCA-SIC as the only predictor. Two different data sets are employed for each target variable to illustrate uncertainty associated with the observations: a land-only station-based data set (the Global Historical Climatology Network-Climate Anomaly Monitoring System, GHCN-CAMS, for surface air temperature; the ENSEMBLES observational data set in Europe, E-OBS, for precipitation) and a land-sea data set including satellite-derived information (the European Centre for Medium-Range Weather Forecasts ERA-Interim reanalysis, ERA-int, for surface air temperature; the Global Precipitation Climatology Project, GPCP, for precipitation). Cross-validated hindcasts of winter surface air temperature yield statistically significant skill in northern Europe, from the British Isles to western Russia, and in central North Africa; robust temperature skill is also found at the southern coast of Greenland (Fig. 3a,b). As expected, the skill pattern tightly projects on the canonical signature of the NAO and largely reflects its impact through 
a

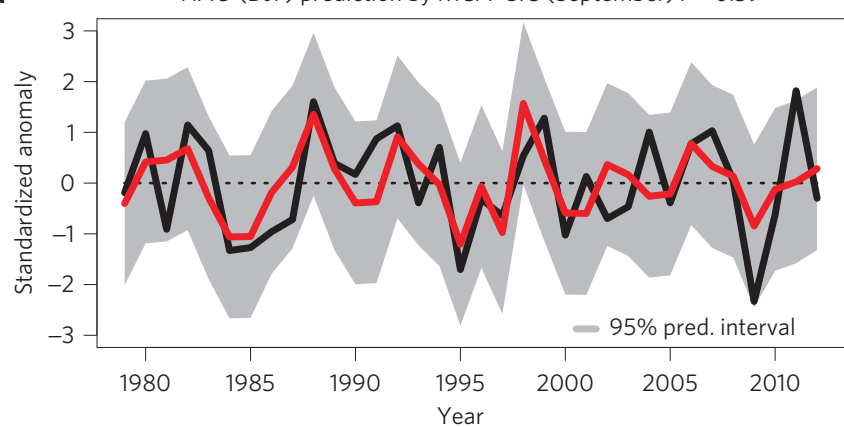

b

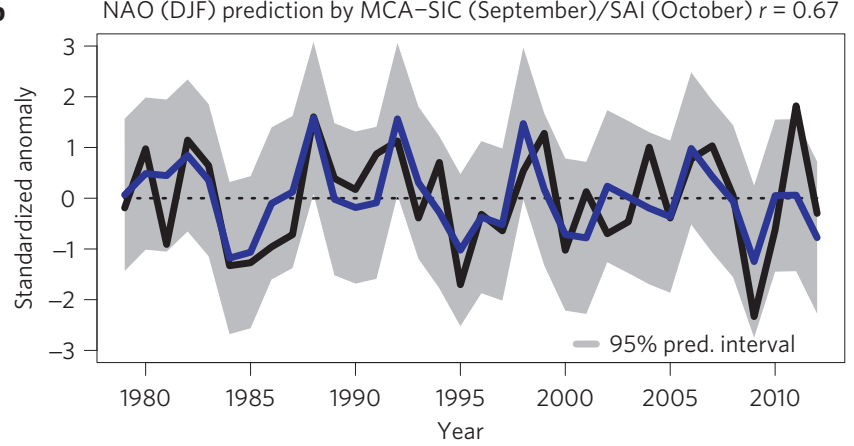

Figure 2 | Cross-validation skill of the NAO. Detrended winter NAO index predicted by the statistical model using a, the September MCA-SIC as the only predictor (red) and $\mathbf{b}$, the combination of the September MCA-SIC and October SAI (blue), with the 95\% prediction interval (grey shading) and from reanalysis (ERA-int; ref. 27; black). The correlation between the observed and predicted time series $(r)$ is indicated atmospheric advection $^{11}$ (Fig. 1b). Cross-validated hindcasts of winter precipitation yield statistically significant skill over the Scandinavian Peninsula and part of the Iberian Peninsula (Fig. 3c,d). Again, the skill pattern resembles the NAO-like dipolar distribution over Europe, which is mostly associated with latitudinal shifts of the North Atlantic storm track ${ }^{11}$. This confirms that there is substantial skill in empirical predictions of the European winter climate from anomalous states of the Arctic sea-ice concentration in September, with a three-month lead time.

The NAO predictability gained from the sea-ice variability can be combined with other statistical or/and dynamical prediction systems to further enhance the forecast skill ${ }^{3,20}$. A high potential predictability of the winter NAO from the October Eurasian snowcover extent (SCE) has been demonstrated, in particular when it is based on the snow advance index ${ }^{9}$ (SAI), which describes the rate of increase during October of the Eurasian SCE. The crossvalidated hindcast of the winter NAO index based on the September MCA-SIC expansion coefficient and the October SAI indeed increases the correlation skill to 0.67 (Fig. 2b), thus explaining $45 \%$ of the interannual winter $\mathrm{NAO}$ variance (compared with 0.51 and $26 \%$ from the SAI alone). Hence, combining the surface predictors improves the NAO skill, but at the expense of reducing by one month the lead time of the empirical predictions. Note that owing to the length of the hindcast period we have used the October SAI from weekly SCE data, but a better performance would be obtained by using the daily SCE data that are available over a more recent period ${ }^{9}$. Regardless, our work shows the marked benefit one could get by including cryospheric variability and, in particular, September Arctic sea ice in prediction systems.

An interesting but barely tackled question is whether the September Arctic sea ice is linked to the October Eurasian snow cover. Previous results indicate that an anomalous sea-ice retreat in September causes an atmospheric warming over the Arctic and a$$
\text { a }
$$

C
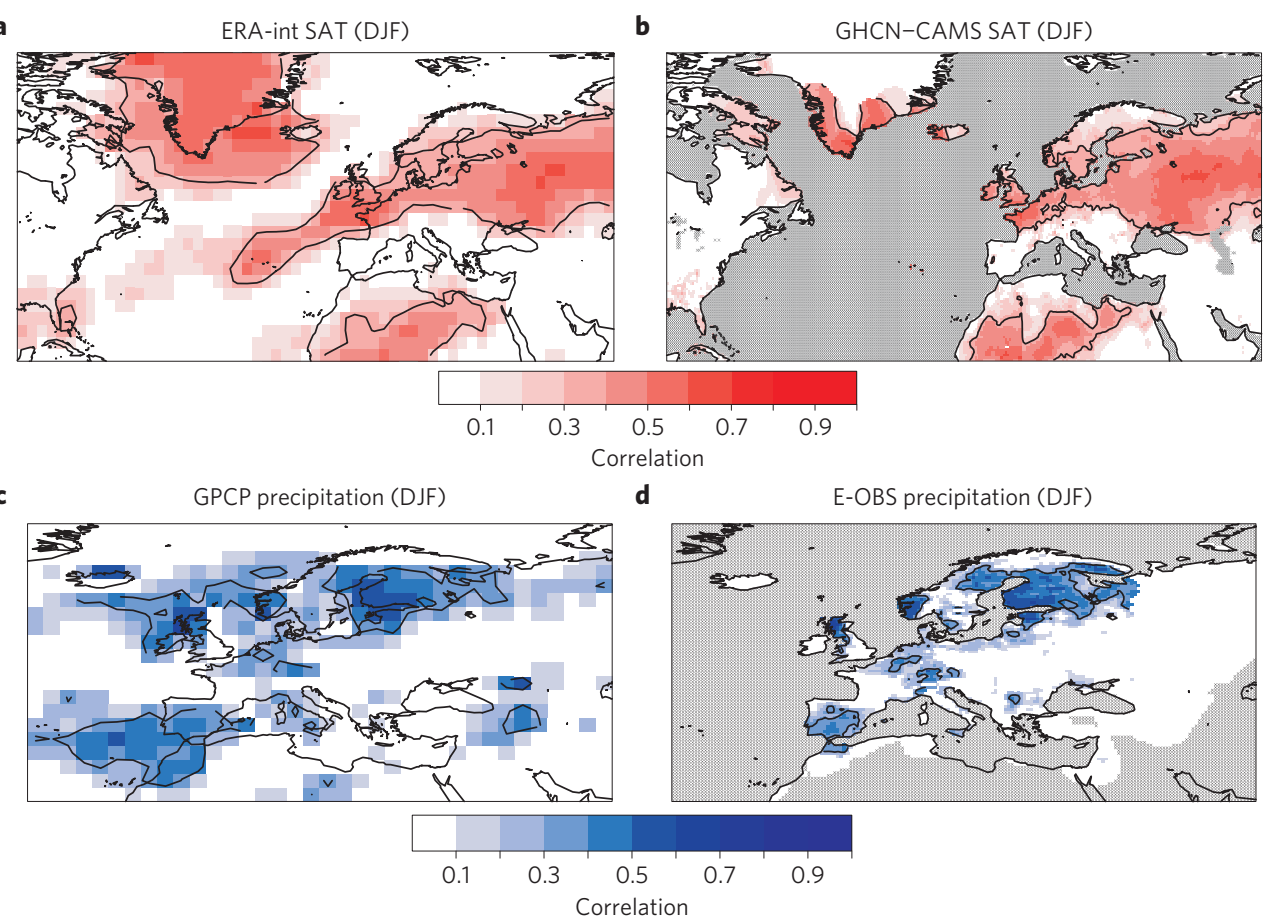

Figure $\mathbf{3}$ | Cross-validation skill of surface climate in Europe. a-d, Correlation maps between the predicted and observational detrended winter surface air temperature (SAT; $\mathbf{a}, \mathbf{b})$ and precipitation anomalies $(\mathbf{c}, \mathbf{d})$; the statistical model uses the September MCA-SIC as the only predictor. The observational data are the land-only station-based GHCN-CAMS (ref. 28) SAT and E-OBS (ref. 29) precipitation, and the land-ocean ERA-int (ref. 27) SAT and GPCP (ref. 30) precipitation. The hatching in $\mathbf{b}, \mathbf{d}$ indicates that no station data are available. Statistically significant areas at $95 \%$ confidence level based on a one-tailed $t$-test (as only positive correlations indicate skill) are contoured. Negative correlations are masked out. 
a $\mathrm{MCA}-\mathrm{SIC}$ (September) $\times$ Z050 (November)

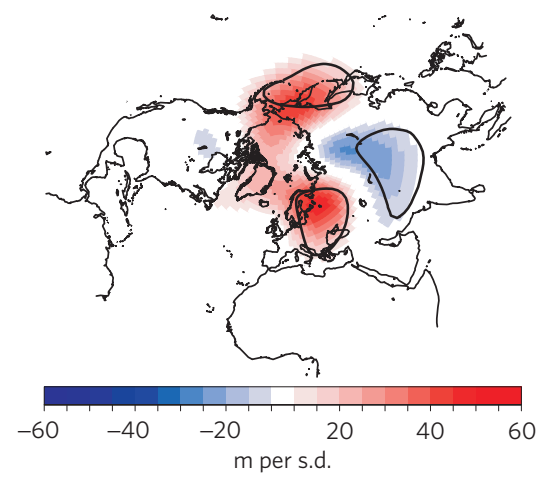

b

MCA-SIC (September) $\times$ Z050 (DJF)

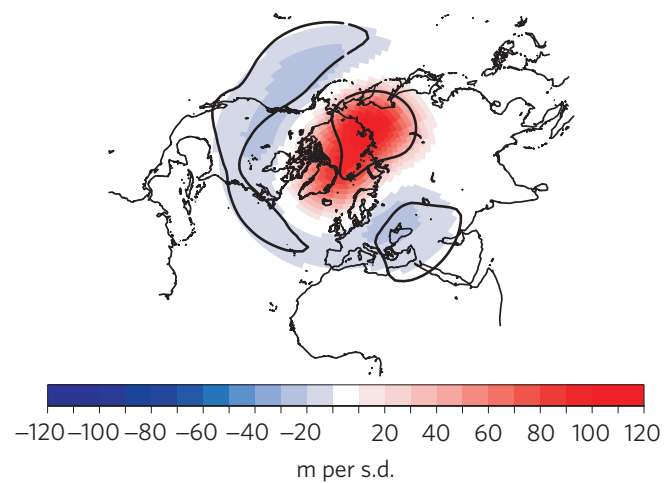

Figure 4 | Stratospheric circulation anomalies associated with the September Arctic sea-ice predictor. a,b, Regression maps, displayed in terms of amplitude ( $\mathrm{m})$, obtained by projecting detrended geopotential height anomalies at $50 \mathrm{hPa}$ in November (a) and winter (b) onto the September MCA-SIC expansion coefficient; shown are patterns associated with the negative NAO phase (Fig. 1b). Statistically significant areas at $95 \%$ confidence level based on a two-tailed $t$-test are contoured.

the adjacent lands, which leads to more moisture availability and enhances precipitation and snowfall over Siberia later in autumn ${ }^{21,22}$. Our linear, empirical approach shows that both surface forcings are related, as the correlation between the MCA-SIC expansion coefficient and the SAI index is 0.45 . This is statistically significant, but it is less than the correlation of each individual predictor with the winter NAO. Hence, September Arctic sea-ice and October Eurasian snow cover seem to represent two distinct predictability sources, albeit related in some way that needs to be investigated.

The mechanism by which September Arctic sea ice influences the winter NAO remains to be identified. Here we hypothesize that it involves the stratosphere, as in the atmospheric response to October Eurasian snow-cover changes ${ }^{9}$. In this case, local snowforced changes in the Siberian High modulate vertical wave activity affecting the polar vortex strength, which eventually leads to downward-propagating perturbations that project on a NAO-like pattern at the surface ${ }^{23}$. The impact of the September Arctic sea ice could involve dynamics on a larger scale in the troposphere, but of similar extent in the stratosphere. Indeed, previous observational ${ }^{24}$ and modelling ${ }^{19}$ evidence indicates that September Arctic sea-ice anomalies, particularly in the Barents-Kara region ${ }^{19}$, are associated with Rossby wavetrain-like circulation anomalies crossing Eurasia later in autumn (that is, November). Such perturbations in the Eurasian sector would then lead a NAO-like pattern (Fig. 1b) by first affecting the vertical propagation of wave activity into the stratosphere. The regression map of geopotential height anomalies at $50 \mathrm{hPa}$ onto the MCA-SIC expansion coefficient indeed shows a strong wavenumber-2 structure in November (Fig. 4a), which is followed by a weakened polar vortex in winter (Fig. 4b). This dynamical framework is the focus of ongoing research, but note that a similar mechanism has been suggested to explain the circulation changes due to the declining trend in the late summer Arctic SIC (ref. 25).

Extended range predictability of surface air temperature and precipitation anomalies, which are the fields that most affect human activities, is linked to our ability to skilfully forecast the boundary conditions that drive the associated atmospheric circulation ${ }^{3}$. The results shown here highlight the need for present dynamical prediction systems to correctly represent and forecast the evolution of interannual Arctic sea-ice variability for improving their skill at predicting the surface winter climate in Europe.

\section{Methods}

Our analysis is based on lag MCA, which is widely used to highlight the influence of the ocean on the atmosphere when the former leads by more than the persistence of the latter ${ }^{7,16}$. Here, the predictor field is autumn Arctic SIC anomalies from the National Oceanic and Atmospheric Administration/National Snow and Ice Data Center passive microwave monthly Northern Hemisphere data $\operatorname{set}^{26}$. The predictand field is SLP anomalies averaged from December to February, over the North Atlantic-European region $90^{\circ} \mathrm{W}-40^{\circ} \mathrm{E}, 20^{\circ} \mathrm{N}-90^{\circ} \mathrm{N}$ from the ERA-int (ref. 27). The same region is used to define the winter NAO index, obtained as the leading principal component of winter SLP anomalies ${ }^{11}$. MCA carries out a singular value decomposition of the covariance matrix between (area-weighted) autumn SIC and winter SLP, and provides a pair of spatial patterns and associated standardized time series (expansion coefficients) for each covariability mode. The MCA results are presented in terms of regression maps (Figs 1 and 4), obtained by projecting the anomaly time series for a given field onto the expansion coefficient associated with the predictor (that is, MCA-SIC).

The statistical prediction model is based on linear regression where the predictand is either the winter NAO index (Fig. 2) or a target surface variable (Fig. 3) and the predictor is the September MCA-SIC expansion coefficient. The statistical prediction model follows a one-year-out cross-validation method, which avoids artificial skill ${ }^{20}$. The SAI (ref. 9) is combined with the September MCA-SIC expansion coefficient to improve the cross-validated hindcast of the NAO index using two predictors. The SAI index is based on weekly Eurasian snow-cover extent in October over the period 1979-2012.

Several independent data sets have been used to assess the cross-validation hindcast skill of surface winter climate in Europe: the GHCN-CAMS (ref. 28) land-only two-metre temperature data set, which is a global monthly high-resolution $\left(0.5^{\circ} \times 0.5^{\circ}\right)$ data set combining the station observations collected from GHCN version 2 with those of CAMS; the E-OBS (ref. 29) version 9 precipitation, which is a European land- only daily high-resolution $\left(0.5^{\circ} \times 0.5^{\circ}\right)$ precipitation data set; the GPCP (ref. 30) version -2.2 data set of the National Aeronautics and Space Administration/Goddard Space Flight Center's Laboratory for Atmospheres, which consists of monthly means of precipitation over land and ocean derived from satellite and gauge measurements on a $2.5^{\circ} \times 2.5^{\circ}$ global grid; and the two-metre temperature from ERA-int (ref. 27) at $2.5^{\circ} \times 2.5^{\circ}$ spatial resolution.

The study covers the 34-year period 1979/1980-2012/2013. All monthly anomalies are calculated by subtracting the corresponding monthly climatology. To reduce the influence of long-term trends, monthly detrended anomalies are considered. As the Arctic SIC evolution since 1979 is not linear ${ }^{24,26}$, a third-order polynomial (that is, cubic trend) estimated by least squares was removed from each variable. We found, however, that linear detrending provides very similar results. Statistical significance of the cross-validated hindcasts (regression maps) is assessed using a one-tailed (two-tailed) Student's $t$-test for correlation at $95 \%$ confidence level. To avoid obtaining too liberal statistical thresholds, we use an effective sample size that takes into account the autocorrelation of the winter NAO index, yielding 25 degrees of freedom.

Received 13 November 2013; accepted 14 February 2014; published online 23 March 2014

\section{References}

1. Rodwell, M. J. \& Doblas-Reyes, F. J. Medium-range, monthly, and seasonal prediction for Europe and the use of forecast information. J. Clim. 19, 6025-6046 (2006). 
2. Doblas-Reyes, F. J. et al. Addressing model uncertainty in seasonal and annual dynamical ensemble forecasts. Q. J. R. Meteorol. Soc. 135, 1538-1559 (2009).

3. Doblas-Reyes, F. J., García-Serrano, J., Lienert, F., Pintó-Biescas, A. \& Rodrigues, L. R. L. Seasonal climate predictability and forecasting: Status and prospects. WIREs Clim. Change 4, 245-268 (2013).

4. Arribas, A. et al. The GloSea4 ensemble prediction system for seasonal forecasting. Mon. Weath. Rev. 139, 1891-1910 (2011).

5. Kim, H-M., Webster, P. J. \& Curry, J. A. Seasonal prediction skill of ECMWF System 4 and NCEP CFSv2 retrospective forecast for the Northern Hemisphere winter. Clim. Dynam. 39, 2957-2973 (2012).

6. Rodwell, M. J. \& Folland, C. K. Atlantic air-sea interaction and seasonal predictability. Q. J. R. Meteorol. Soc. 128, 1413-1443 (2002).

7. Czaja, A. \& Frankignoul, C. Observed impact of Atlantic SST anomalies on the North Atlantic Oscillation. J. Clim. 15, 606-623 (2002).

8. Folland, C. K., Scaife, A. A., Lindesay, J. \& Stephenson, D. B. How potentially predictable is northern European winter climate a season ahead? Int. J. Climatol. 32, 801-818 (2012).

9. Cohen, J. \& Jones, J. A new index for more accurate winter predictions. Geophys. Res. Lett. 38, L21701 (2011).

10. Brands, S., Manzanas, R., Gutiérrez, J. M. \& Cohen, J. Seasonal predictability of wintertime precipitation in Europe using the snow advance index. J. Clim. 25, 4023-4028 (2012)

11. Hurrell, J. W., Kushnir, Y., Visbeck, M., Ottersen, G. in An overview of the North Atlantic Oscillation (eds Hurrell, J. et al.) 1-35 (AGU Geophys. Monogr. Vol. 134, 2003).

12. Scaife, A. A., Folland, C. K., Alexander, L. V., Moberg, A. \& Knight, J. R. European climate extremes and the North Atlantic Oscillation. J. Clim. 21, $72-83$ (2008).

13. Jeong, J-H. et al. Impacts of snow initialization on subsesonal forecasts of surface air temperature for the cold season. J. Clim. 26, 1956-1972 (2013).

14. Orsolini, Y. J. et al. Impact of snow initialization on sub-seasonal forecasts. Clim. Dynam. 41, 1969-1982 (2013).

15. Riddle, E. E., Butler, A. H., Furtado, J. C., Cohen, J. \& Kumar, A. CFSv2 ensemble prediction of the winter Arctic Oscillation. Clim. Dynam. 41, 1099-1116 (2013).

16. Wu, Q. \& Zhang, X. Observed forcing-feedback processes between Northern Hemisphere atmospheric circulation and Arctic sea ice coverage. Geophys. Res. Lett. 115, D14119 (2010).

17. Li, F. \& Wang, H. Autumn sea ice cover, winter Northern Hemisphere Annular Mode, and winter precipitation in Eurasia. J. Clim. 26, 3968-3981 (2013).

18. Tang, Q., Zhang, X., Yang, X. \& Francis, J. A. Cold winter extremes in northern continents linked to Arctic sea ice loss. Environ. Res. Lett. 8, 014036 (2013).

19. Honda, M., Inoue, J. \& Yamane, S. Influence of low Arctic sea-ice minima on anomalously cold Eurasian winters. Geophys. Res. Lett. 36, L08707 (2009).

20. Coelho, C. A. S., Pezzulli, S., Balmaseda, M., Doblas-Reyes, F. J. \& Stephenson, D. B. Forecast calibration and combination: A simple bayesian approach for ENSO. J. Clim. 17, 1504-1516 (2004).
21. Ghatak, D. et al. Simulated Siberian snow cover response to observed Arctic sea ice loss, 1979-2008. J. Geophys. Res. 117, D23108 (2012).

22. Cohen, J., Furtado, J. C., Barlow, M. A., Alexeev, V. A. \& Cherry, J. E. Arctic warming, increasing snow cover and widespread boreal winter cooling. Environ. Res. Lett. 7, 014007 (2012).

23. Cohen, J., Barlow, M., Kushner, P. J. \& Saito, K. Stratosphere-troposphere coupling and links with Eurasian land surface variability. J. Clim. 20, 5335-5343 (2007).

24. Francis, J. A., Chan, W., Leathers, D. J., Miller, J. R. \& Veron, D. E. Winter Northern Hemisphere weather patterns remember summer Arctic sea-ice extent. Geophys. Res. Lett. 36, L07503 (2009).

25. Jaiser, R., Dethloff, K. \& Handorf, D. Stratospheric response to Arctic sea ice retreat and associated planetary wave propagation changes. Tellus A 65, 19375 (2013)

26. Comiso, J. C. Bootstrap sea ice concentrations from Nimbus-7 SMMR and DMSP SSM/I-SSMIS - version 2 Boulder, Colorado USA: NASA DAAC (National Snow and Ice Data Center, 2000, updated 2012); http://nsidc.org/data/nsidc-0079.html.

27. Dee, D. P. et al. The ERA-Interim reanalysis: Configuration and performance of the data assimilation system. Q. J. R. Meteorl. Soc. 137, 553-597 (2011).

28. Fan, Y. \& van den Dool, H. A global monthly land surface air temperature analysis for 1948-present. J. Geophys. Lett. 113, D01103 (2008).

29. Haylock, M. R. et al. A European daily high-resolution gridded data set of surface temperature and precipitation for 1950-2006. J. Geophys. Res. 113, D20119 (2008)

30. Adler, R. F. et al. The version 2 Global Precipitation Climatology Project (GPCP) monthly precipitation analysis (1979-present). J. Hydrometeor. 4, 1147-1167 (2003).

\section{Acknowledgements}

We are grateful to J. Cohen (AER Inc., Lexington, USA) for kindly providing the SAI and to F. J. Doblas-Reyes (IC3, Barcelona, Spain) for discussions. The research leading to these results has received financial support from the European Union seventh Framework Programme (FP7 2007-2013), under grant agreement no.308299 (NACLIM, see www.naclim.eu).

\section{Author contributions}

J.G-S. led the analysis. C.F. discussed the results and co-wrote the manuscript.

\section{Additional information}

Reprints and permissions information is available online at www.nature.com/reprints. Correspondence and requests for materials should be addressed to J.G-S.

\section{Competing financial interests}

The authors declare no competing financial interests. 


\title{
RETRACTION
}

\section{Retraction: High predictability of the winter Euro-Atlantic climate from cryospheric variability}

\author{
J. García-Serrano and C. Frankignoul
}

Nature Geoscience http://dx.doi.org/10.1038/ngeo2118; published online 23 March 2014; retracted 14 April 2014

In our Letter, a conceptual error in the cross-validation approach led to an overestimation of the predictive skill of the winter (December-February) North Atlantic Oscillation (NAO) and Euro-Atlantic surface climate from Arctic sea-ice variability. The error does not affect the hindcasts based on the snow advance index.

Specifically, to produce the one-year-out cross-validated hindcasts based on Arctic sea-ice variability, we performed a maximum covariance analysis (MCA) between Arctic sea-ice concentration (SIC) anomalies and winter Euro-Atlantic sea-level pressure anomalies in the period 1979/80 to 2012/13. We then applied one-year-out cross-validation using subsets of years from the SIC time series derived from the whole period. Thereby, the regression coefficients (that is, slope and intercept) and predictor value of the statistical model were estimated assuming the knowledge of the MCA fields in the year out. This procedure overestimates the cross-validation skill.

One-year-out cross-validated hindcasts instead require cross-validation of the MCA pattern-generation in the year out, thus performing an MCA on the remaining years. Following this approach, the cross-validated skill in hindcasting the winter NAO index using September SIC over the whole Arctic is 0.08 , indicating that there is no predictive skill from Arctic sea-ice variability. The cross-validated NAO skill using October or November SIC over the whole Arctic is 0.22 and 0.18 , respectively, suggesting some skill.

Although our analysis reveals no skill in sea-ice-based NAO predictions with three months lead time, the limited skill from October-November sea-ice concentration supports the notion that sea-ice information should be incorporated in dynamical prediction systems to improve their skill at forecasting the surface winter climate in Europe.

Following the identification of the error in our cross-validation approach, this Letter has been retracted. We are grateful to Geert Jan van Oldenborgh (KNMI, De Bilt, The Netherlands) for identifying this error. We also thank Francisco J. Doblas-Reyes (IC3, Barcelona, Spain) for discussions. 